Finally, as society's expectations of professionals become even higher, there must be greater understanding for doctors and the risks that they personally take when dealing every day with the pain and suffering of others. As a minimum, patients' rights should surely extend to doctors when they themselves are patients, and healthcare professionals must acknowledge the fact that healers, when they are ill, may also suffer profoundly. They need healing too, not just by other experts, but by the understanding and compassion of their colleagues.

As the principal conditions potentially impairing doctors' fitness to practise are mental health problems, and alcohol-related and drug-related problems, psychiatrists have an important role to play, both in the destigmatisation of mental illness and by providing appropriate assessment and treatment. The $\mathrm{N}$ ational Clinical Assessment Authority (N CAA, 2004) in the UK comprehensively review ed health-related factors involved in doctors' performance as well as the personal characteristics and behaviour of doctors.

\section{References}

Bennett, J. \& O'Donovan, D. (2001) Substance misuse by doctors, nurses and other healthcare workers. Current Opinion in Psychiatry, 14, 195-199.

Burbeck, R., Coomber, S., Robinson, S. M., et al (2002) 0 ccupational stress in consultants in accident and emergency medicine: a national survey of levels of stress at work. Emergency Medicine Journal, 19, 234-238.

Ghodse, H. (2000) Doctors and their health - who heals the healers? In Doctors and Their Health (eds H. Ghodse, S. Mann \& P. Johnson), pp. 10-14. Sutton: Reed Healthcare Ltd.

National Clinical Assessment Authority (2004) Understanding Performance: Difficulties in Doctors. London: NCAA.

Visser, M., Smets, E. M., O ort, F. J., et al (2003) Stress, satisfaction and burnout among Dutch medical specialists. Canadian Medical Association Journal, 168, 271-275.

Winter, R. 0. \& Birnberg, B. (2002) Working with impaired residents: trials, tribulations and successes. Family Medicine, 34, 190-196.

\title{
Women's mental health in a context of violence, exploitation and oppression
}

\author{
David Skuse
}

Behavioural and Brain Sciences Unit, Institute of Child Health, London WC1 1EH, UK, email d.skuse@ich.ucl.ac.uk

n this issue we address the sensitive issue of societal attitudes towards women, in particular violence, exploitation and oppression, and their consequences for women's mental health. The subject of exploitation and abuse of women, by men, is rarely out of the headlines. Yet the prevalence of the problem does not seem to diminish, despite widespread publicity. In their fascinating review of the confluence of partner violence and substance misuse with mental health problems, Cari Jo Clark and $\mathrm{Grace}$ W yshak estimate that up to two-thirds of women are subject to such violence, extrapolating from studies conducted in a wide variety of countries and cultures. They make the point that substance misuse and mental health disorders are both risk factors for such behaviour and outcomes of it.

Psychiatric interventions to mitigate the effects of violence upon women's mental health must be sensitive to local cultural contexts, but there are formidable complexities. These are exemplified in the contribution from D rs Lari, Alaghehbandan and Joghataei, on the psychosocial and cultural motivations for self-inflicted burns among Iranian women. They provide an insight into aspects of a social phenomenon that is truly disturbing: the rising incidence of self-immolation among Iranian women, especially during the early years of marriage. There is a supposition that in many cases this course of action is taken to escape from a violent relationship, or one in which the woman is exploited by traditional male values, but the truth is we cannot be sure. In many cases the women concerned are killed by their actions; in others they deny intent and ascribe the burns to an accident.

Finally, D rs Medina-Mora and Lara have contributed a review of attitudes to women and their mental health in Mexico, a culture where the term 'machismo' still holds meaning. Mexican society has, by this account, clear divisions into what are regarded as appropriate male and female roles. Yet, as women in many countries in the Western world have found following their 'liberation' in the 1960s, all to o often the freedom to jo in a male-dominated w orld of work means dual responsibilities and limited opportunities to advance along a parallel path to men outside the home. In 
M exico, there is an additional twist to the differentiation of gender roles, in the sense that women are expected not to drink alcohol, because it is not compatible with their domestic responsibilities. And so we come back to the issue of domestic violence which was raised by Clark and Wyshak, with the misuse of alcohol by men being closely correlated with physical violence against female partners.

Psychiatrists around the world need to be alert to the continuing stresses on w omen, from family, partners, work and many other obligations; cultural influences on female mental health are rarely trivial.

\title{
The confluence of violence towards an intimate partner, substance misuse and mental health: a worldwide problem affecting women
}

\author{
Cari Jo Clark, MPH ScD ${ }^{1}$ and Grace Wyshak, $\mathrm{PhD}^{2}$ \\ ${ }^{1}$ Department of Population and International Health, Harvard School of Public Health, Boston, Massachusetts, \\ USA \\ ${ }^{2}$ Associate Professor, Department of Psychiatry, Harvard Medical School, and Departments of Biostatistics \\ and Population and International Health, Harvard School of Public Health, 665 Huntington Avenue, Boston, \\ MA 02115, USA, email wyshak@hsph.harvard.edu
}

$V$ iolence towards an intimate partner, substance misuse and other mental health disorders are problems that tend to cluster together and result in multiple burdens for afflicted individuals (Desjarlais et al, 1995; Wyshak \& Modest, 1996; Wyshak, 2000). They are prevalent not only in high-risk groups but also among members of the general public seeking primary healthcare (Bauer et al, 2000; World Health O rganization, 2001), where their afflictions often go undiagnosed and untreated (Edlund et al, 2004; Kramer et al, 2004). Furthermore, violence towards an intimate partner, substance misuse and other mental health disorders involve common symptom pathways, such as psychiatric distress, headache, abdominal pain, gastrointestinal problems and multiple somatic complaints (Berwick et al, 1991), which suggests that the use of an integrated set of screening instruments may lead to early detection and treatment for patients who are suffering from one or more of these problems.

\section{The role of gender}

Violence directed at an intimate partner disproportionately affects women: between $10 \%$ and $69 \%$ of women have at some time experienced such violence, according to a review of 48 population-based studies from a variety of countries around the world (Krug et al, 2002). Similarly important, tobacco smoking, alcohol consumption and the use of illicit drugs (excluding ecstasy, solvents and cannabis) cause a total of approximately 6.9 million deaths annually: 4.9, 1.8 and 0.2 million, respectively (World Health 0 rganization, 2002). Although men use these substances at higher rates than women, the health impact for women is no less significant and for some substances may be even greater (Cormier et al, 2004). Furthermore, neuropsychiatric disorders as a whole afflict approximately 450 million individuals worldwide (World Health $O$ rganization, 2001). Of these disorders, depression, which disproportionately affects women, is the leading cause of disability worldwide (World $H$ ealth 0 rganization, 2001).

\section{Confluence of the three types of problem}

Violence towards an intimate partner, substance misuse and other mental health diso rders are highly correlated with each other. Substance misuse and other mental health disorders are risk factors for and health outcomes of violence perpetrated by an intimate partner (Riggs et al, 2000). Substance misuse and other mental disorders themselves are highly correlated under the rubric of dual diagnosis. Research specifically addressing these interconnections has found high correlations between the experience of violence, substance misuse and psychiatric distress, characterised by gender and ethnic differences; notably, in women depression and alcoholism are highly correlated (W yshak \& Modest, 1996).
Violence directed at an intimate partner disproportionately affects women: between $10 \%$ and $69 \%$ of women have at some time experienced such violence, according to a

review of 48 population-based

studies from a variety of

countries around the world. 